\title{
Helicobacter pylori infection promotes the invasion and metastasis of gastric cancer through increasing the expression of matrix metalloproteinase-1 and matrix metalloproteinase-10
}

\author{
HAO JIANG ${ }^{1}$, YUJUAN ZHOU ${ }^{2}$, QIANJIN LIAO ${ }^{2}$ and HONGJUAN OUYANG ${ }^{1}$ \\ ${ }^{1}$ Gastric Cancer Research Center of Hunan, Department of Oncology, The First Affiliated Hospital, University of South China, \\ Hengyang, Hunan 421001; ${ }^{2}$ Hunan Cancer Hospital and the Affiliated Cancer Hospital of Xiangya School of Medicine, \\ Central South University, Changsha, Hunan 410013, P.R. China
}

Received December 9, 2013; Accepted May 9, 2014

DOI: $10.3892 /$ etm.2014.1822

\begin{abstract}
The aim of this study was to investigate the effect and mechanism of Helicobacter pylori infection in the invasion and metastasis of gastric cancer. Specimens from 80 patients with gastric cancer (of which 20 patients had metastatic gastric cancer) and 40 patients with chronic gastritis were included in this study. H. pylori infection was determined by ELISA and the expression of matrix metalloproteinase-1 (MMP-1) and MMP-10 was observed using immunohistochemistry. The correlation between $H$. pylori infection and the clinical pathological features of gastric cancer was analyzed by SPSS 13.0 software. The protein expression levels of MMP-1 and MMP-10 in MGC-803 cells infected with $H$. pylori were analyzed using western blotting. $H$. pylori infection was found in 62 of the 80 patients with gastric cancer and in 13 of the 40 patients with chronic gastritis. In addition, H. pylori infection was correlated with the staging and lymph node metastasis, but not with the gender, age and histological types of patients. $H$. pylori infection was also significantly correlated with the expression of MMP-1 and MMP-10 ( $\mathrm{r}=0.8718, \mathrm{P}<0.05$ and $\mathrm{r}=0.5477, \mathrm{P}<0.05$, respectively). The expression of MMP-1 and MMP-10 was significantly upregulated following induction by $H$. pylori infection $(\mathrm{P}<0.05)$, with significant effects occurring following infection for 12 and $6 \mathrm{~h}$, respectively. H. pylori infection may promote the invasion and metastasis of gastric cancer by increasing the expression of MMP-1 and MMP-10.
\end{abstract}

Correspondence to: Dr Hao Jiang, Gastric Cancer Research Center of Hunan, Department of Oncology, The First Affiliated Hospital, University of South China, 69 Chuanshan Road, Hengyang, Hunan 421001, P.R. China

E-mail: jhao16859@126.com

Key words: gastric cancer, Helicobacter pylori, matrix metalloproteinase-1, matrix metalloproteinase-10, metastasis

\section{Introduction}

The development of gastric cancer involves several factors, among which Helicobacter pylori infection is the most important (1). H. pylori is a type of micro-aerobic, Gram-negative, spiral bacterium, which resides between the gastric mucous layer and the gastric surface. H. pylori secretes urease, which damages the gastric mucosal barrier. The $H$. pylori lipopolysaccharide inhibits the binding of laminin to its receptor, resulting in gastric mucosal injury (2). The vacuolating toxin gene of $H$. pylori can change ion permeability, leading to cell degeneration (3), and damage the gastric mucosa, causing erosion or ulceration. H. pylori expresses cytotoxin-associated gene (Cag) A, which generates a cytotoxic effect and induces inflammatory and immune responses. The deformation and necrosis of mucosal cells and inflammatory infiltration can be observed in $H$. pylori-infected lesions, and specific antibodies can be detected in serum $(4,5)$.

Matrix metalloproteinases (MMPs) are highly homologous, zinc-dependent endopeptidases that can degrade the basement membrane. To date, 19 types of MMPs have been identified. All MMPs are secreted by mesenchymal cells in the form of a protease precursor, and can be inhibited by tissue inhibitors of metalloproteinases (TIMPs). It has been found that MMPs are important in tumor invasion, metastasis, cardiovascular disease and diabetes, and are closely associated with the degradation of the extracellular matrix (ECM) of tumor cells (6).

Invasion and metastasis are the prominent features of malignant tumors, and the degradation of the ECM is one of the key steps involved in these processes. Matrix degradation primarily depends on proteolytic enzymes. An increasing number of studies have shown that the invasion and metastasis of tumor cells are closely associated with MMP production. In vitro and in vivo studies have shown that MMPs are functional in various stages of tumor progression, influencing tumor genesis, growth, angiogenesis and metastasis (7-9). The interaction of MMPs with the basement membrane is the initiating signal of tumor invasion and metastasis.

As mentioned above, tumor invasion and metastasis involve the secretion of MMPs. MMP-1 predominantly degrades the stroma, which is closely associated with local 
invasion and metastasis $(10,11)$. MMP-10 is considered to be the most important factor in human tumor cells that can activate other MMP (such as MMP-1) precursors (12). MMP-10 can also degrade certain interstitial proteins, contributing to metastasis (13).

In this study, in order to determine the effect of $H$. pylori infection on gastric cancer, the associations among $H$. pylori infection, the expression of MMP-1 and MMP-10 and the invasion and metastasis of gastric cancer were investigated. H. pylori infection and MMP-1 and MMP-10 expression were detected in gastric cancer and chronic gastritis specimens. The association between $H$. pylori infection and the clinical pathological features of gastric cancer were then analyzed. Additionally, the expression of MMP-1 and MMP-10 following H. pylori infection in MGC-803 cells was studied.

\section{Materials and methods}

Cell line and reagents. The MGC-803 human gastric cancer cell line, a poorly differentiated gastric adenocarcinoma, was provided by the Department of School of Biological Science, Shandong Normal University (Jinan, China) and preserved in the Central Laboratory of the First Affiliated Hospital of Nanhua University (Hengyang, China). MGC-803 cells were cultured in high-glucose Dulbecco's modified Eagle's medium (DMEM) supplemented with $10 \%$ fetal bovine serum, $100 \mathrm{U} / \mathrm{ml}$ penicillin and $100 \mathrm{mg} / \mathrm{ml}$ streptomycin at $37^{\circ} \mathrm{C}$ in a humidified incubator with $5 \% \mathrm{CO}_{2}$.

A Streptavidin-peroxidase (SP) immunohistochemistry kit, hematoxylin stain and 3,3' diaminobenzidine (DAB) chromogenic agent were all purchased from Fuzhou Maixin Biotechnology Development Co., Ltd. (Fuzhou, China). Mouse anti-human MMP-1 and MMP-10 monoclonal antibodies for immunohistochemistry and western blotting were purchased from Santa Cruz Biotechnology, Inc. (Santa Cruz, CA, USA). Anti-mouse immunoglobulin G (IgG) was purchased from Boster Biological Technology, Ltd. (Wuhan, China). The ELISA kit was obtained from Bio-Check, Inc. (Foster City, CA, USA) and the bicinchoninic acid assay reagent was purchased from Pierce Chemical Co. (Rockford, IL, USA).

Patient samples. Between 2005 and 2009, 80 cases of surgically resected and pathologically diagnosed gastric cancer paraffin specimens from the First Affiliated Hospital of Nanhua University were collected. The patients with gastric cancer were aged between 23 and 78 years, with a mean age of $53.2 \pm 12.7$ years, and included 58 males and 22 females. Prior written and informed consent was obtained from every patient and the study was approved by the Ethics Review Board of Nanhua University. Of the 80 cases of gastric cancer, there were 20 cases of highly differentiated carcinoma, 20 cases of moderately differentiated carcinoma and 40 cases of poorly differentiated carcinoma. A total of 20 cases of gastric cancer exhibited lymph node metastasis. With regard to the extent of infiltration, there were 15 cases of early gastric cancer with gastric mucosal tissue infiltration limited to the mucosa or submucosal layer (regardless of lymph node metastasis) and 65 cases of advanced gastric cancer. Forty specimens of chronic superficial gastritis were collected as the control group. All specimens were fixed in formalin and embedded in paraffin. Specimens were sliced into $5-\mu \mathrm{m}$ sections.

Infection of MGC-803 cells with H. pylori. A total of $1 \times 10^{5}$ MGC-803 cells were seeded in T75 tissue culture flask and $6 \mathrm{ml}$ serum-free DMEM was added. After $24 \mathrm{~h}$ of synchronization, the culture medium was discarded. H. pylori culture medium was then added. According to the method described in a previous study (4), bacteria density was adjusted to $1 \times 10^{8} \mathrm{CFU} / \mathrm{ml}$, and two-fold diluted with serum-free DMEM. The MGC-803 cells were then co-cultured with $H$. pylori for $6,12,24$ and $48 \mathrm{~h}$.

ELISA. Fasting blood ( $2 \mathrm{ml})$ was collected from all patients. For H. pylori IgG detection, ELISA was performed according to the manufacturer's instructions (Bio-Check, Inc.). H. pylori IgG levels $>20 \mathrm{U} / \mathrm{ml}$ were considered to be a positive result.

Immunohistochemistry. Immunohistochemical staining (the SP method) was conducted to detect the protein expression of MMP-1 and MMP-10 in gastric cancer and chronic gastritis samples. Briefly, the samples were fixed in formaldehyde and embedded in paraffin. The sections were dewaxed, rehydrated in graded alcohols and processed prior to incubation with antibodies. Subsequent to blocking, the sections were incubated with primary antibodies at $37^{\circ} \mathrm{C}$ in the dark for $1 \mathrm{~h}$. Secondary antibodies were then added and incubated in dark for 30 min following washing with phosphate-buffered saline (PBS). The sections were then developed with DAB chromogenic reagent and counterstained with hematoxylin. The working concentration of MMP-1 and MMP-10 primary antibody was 1:200. PBS was used in the negative control group instead of primary antibody. Positive staining for MMP-1 and MMP-10 was located in the cytoplasm. A total of $\geq 10$ high-power fields were randomly selected (magnification, $\mathrm{x} 200$ ), and $\geq 1,000$ cells were counted. The staining intensity and percentage of positive cells was scored in each slice. Staining intensity was defined as 0 points for no staining, 1 point for pale yellow, 2 points for brownish yellow and 3 points for tan. The positive staining cell ratio was defined as 0 points for no staining, 1 point for $<30 \%, 2$ points for $30-60 \%$ and 3 points for $>60 \%$. The combined points total for the staining intensity and positive staining ratio was furthermore defined as follows: $0-2$ points, weak positive or negative; $3-4$ points, positive; and 5-6 points, strong positive. The rate of positive staining was calculated using the following formula: Positive rate $=($ weak positive case number + strong positive case number) / total cases x $100 \%$.

Western blotting. At 6, 12, 24 and $48 \mathrm{~h}$ after $H$.pylori infection, total proteins were extracted from MGC- 803 cells and separated using SDS-PAGE. The proteins were then transferred onto a nitrocellulose membrane. Subsequent to blocking with non-fat milk, the membrane was incubated with monoclonal mouse anti-MMP-1 and -MMP-10 primary antibodies overnight at $4^{\circ} \mathrm{C}$. The membrane was then washed and incubated with the anti-mouse $\operatorname{IgG}$ secondary antibody at $37^{\circ} \mathrm{C}$ for $1 \mathrm{~h}$, prior to being developed using enhanced chemiluminescence plus reagent (Amersham, GE Healthcare Life Sciences, Piscataway, NJ, USA). $\beta$-actin was used as an internal control. 
Table I. MMP-1 and MMP-10 expression in specimens of gastric cancer and chronic gastritis.

Immunohistochemical score

Type of MMP and

specimen

Cases (n) Weak positive, 0-2 (n)

Positive, 3-4 (n)

Strong positive, 5-6 (n)

P-value

MMP-1

Gastric cancer

80

20

40

Chronic gastritis

MMP-10

Gastric cancer

Metastatic gastric cancer

Chronic gastritis
80

20

40
17

1

30

13

1

28
19

1

9

19

2

10
44

1

48

17

2 $0.004^{\mathrm{a}}$

$<0.001^{\mathrm{b}}$

$<0.001^{\mathrm{c}}$

$0.036^{\mathrm{a}}$

$<0.001^{\mathrm{b}}$

$<0.001^{\mathrm{c}}$

Values of $\mathrm{P}<0.05$ were determined using a one-way analysis of variance least significant difference test: ${ }^{\mathrm{a} G a s t r i c}$ cancer versus metastatic gastric cancer; ${ }^{b}$ metastatic gastric cancer versus chronic gastritis and ${ }^{c}$ gastric cancer versus chronic gastritis. MMP, matrix metalloproteinase.

A

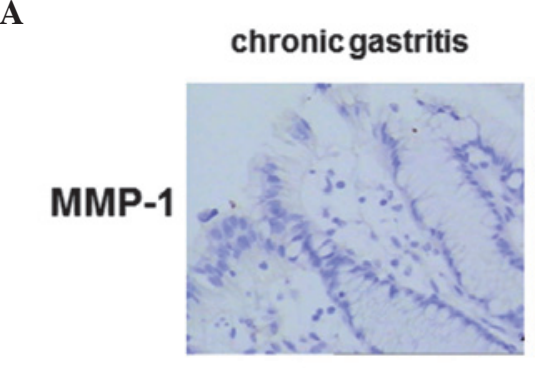

B

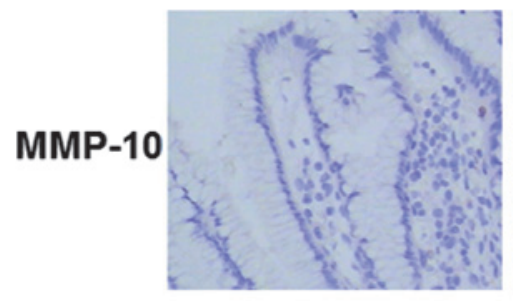

poorly differentiated moderately differentiated gastric cancer
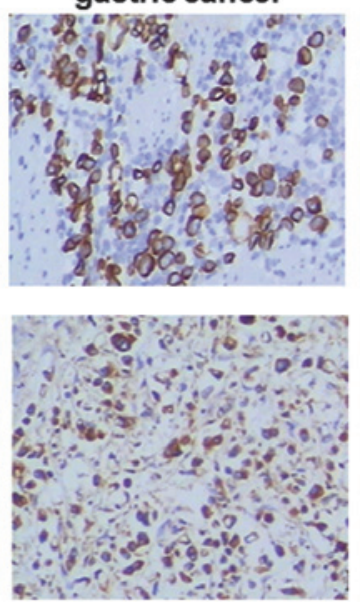
gastric cancer
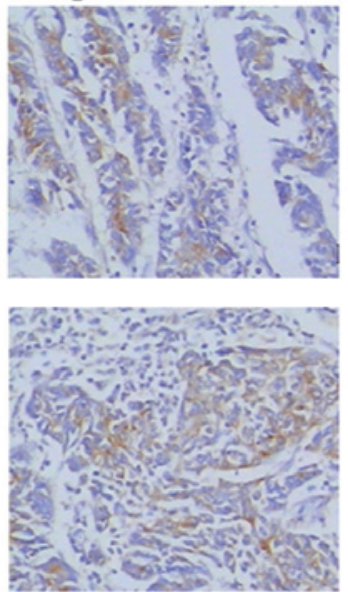

gastric cancerwith lymph node metastasis
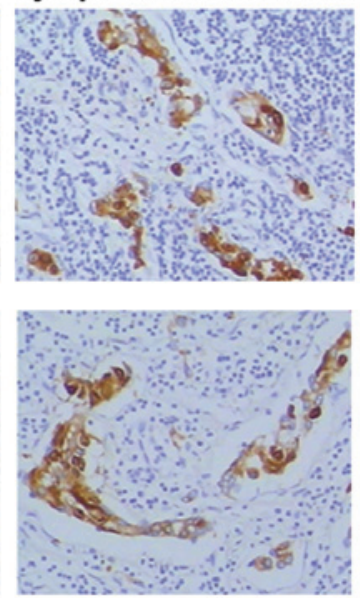

Figure 1. Immunohistochemical analysis of (A) MMP-1 and (B) MMP-10 protein expression in gastric cancer and chronic gastritis tissue. The expression of MMP-1 and MMP-10 was detected by immunohistochemical staining in tissues of chronic gastritis, poorly and moderately differentiated gastric cancer and gastric cancer with lymph node metastasis. Representative immunohistochemical staining images are shown (magnification, x200). MMP, matrix metalloproteinase.

Statistical analysis. SPSS 13.0 (SPSS, Inc., Chicago, IL, USA) software was used for statistical analysis. The immunohistochemical results were compared using a $\chi^{2}$ test. The Spearman's rho correlation test was used to evaluate the correlations between $H$. pylori infection and MMP-1 and MMP-10 expression in gastric cancer. $\mathrm{P}<0.05$ was considered statistically significant.

\section{Results}

MMP-1 and MMP-10 protein expression in gastric cancer is higher than that in chronic gastritis. To determine MMP-1 and MMP-10 protein expression in gastric cancer and chronic gastritis, immunohistochemical staining was performed. Representative immunohistochemical staining results are shown in Fig. 1 and quantitative results are shown in Table I. As shown in Fig. 1, MMP-1 and MMP-10 expression was not detected in the chronic gastritis specimens. However, positive staining for MMP-1 and MMP-10 was detected in the poorly and moderately differentiated gastric cancer and gastric cancer with lymph node metastasis specimens. The positive staining rate was calculated as described in the Materials and methods section. As shown in Table I, the MMP-1-positive rate in patients with gastric cancer, metastatic gastric cancer and chronic gastritis was $78.8 \%(63 / 80), 95.0 \%(19 / 20)$ and $25.0 \%$ (10/40), respectively. The MMP-10-positive rate in the patients with gastric cancer, metastatic gastric cancer and chronic gastritis was $83.4 \%$ (67/80), 95.0\% (19/20) and $30.0 \%$ (12/40), respectively. Statistically, the expression levels of MMP-1 and MMP-10 were significantly higher in the gastric cancer specimens than those in the chronic gastritis specimens $(\mathrm{P}<0.05)$. Furthermore, compared with levels in the gastric cancer specimens, the expression levels of MMP-1 and MMP-10 in the metastatic gastric cancer specimens 
Table II. Correlation analysis of Helicobacter pylori infection, MMP-1 and MMP-10 protein expression and gastric cancer pathological characteristics.

\begin{tabular}{|c|c|c|c|c|c|c|c|c|c|c|}
\hline \multirow[b]{2}{*}{ Parameter } & \multirow[b]{2}{*}{$\begin{array}{c}\text { Cases } \\
\text { (n) }\end{array}$} & \multicolumn{2}{|c|}{ H.pylori infection } & \multirow[b]{2}{*}{ P-value } & \multicolumn{2}{|c|}{ MMP-1 expression } & \multirow[b]{2}{*}{ P-value } & \multicolumn{2}{|c|}{ MMP-10 expression } & \multirow[b]{2}{*}{ P-value } \\
\hline & & $\begin{array}{c}\text { Positive } \\
\text { (n) }\end{array}$ & $\begin{array}{l}\text { Negative } \\
\text { (n) }\end{array}$ & & $\begin{array}{c}\text { Positive } \\
\text { (n) }\end{array}$ & $\begin{array}{c}\text { Negative } \\
\text { (n) }\end{array}$ & & $\begin{array}{c}\text { Positive } \\
\text { (n) }\end{array}$ & $\begin{array}{c}\text { Negative } \\
\text { (n) }\end{array}$ & \\
\hline Age in years & & & & 0.217 & & & 0.432 & 67 & 13 & 0.157 \\
\hline$\geq 50$ & 61 & 49 & 12 & & 48 & 13 & & 53 & 8 & \\
\hline$<50$ & 19 & 13 & 6 & & 15 & 4 & & 14 & 5 & \\
\hline Gender & & & & 0.597 & & & 0.532 & & & 0.098 \\
\hline Male & 58 & 45 & 13 & & 46 & 12 & & 51 & 7 & \\
\hline Female & 22 & 17 & 5 & & 17 & 5 & & 16 & 6 & \\
\hline Lymph node metastasis & & & & $<0.001^{\mathrm{a}}$ & & & $<0.001^{\mathrm{a}}$ & & & $<0.001^{\mathrm{a}}$ \\
\hline With & 52 & 48 & 4 & & 48 & 4 & & 50 & 2 & \\
\hline Without & 28 & 14 & 14 & & 15 & 13 & & 17 & 11 & \\
\hline Clinical stage & & & & $<0.001^{\mathrm{a}}$ & & & $<0.001^{\mathrm{a}}$ & & & $<0.001^{\mathrm{a}}$ \\
\hline Early & 15 & 4 & 11 & & 5 & 10 & & 7 & 8 & \\
\hline Middle & 65 & 58 & 7 & & 58 & 7 & & 60 & 5 & \\
\hline Differentiation degree & & & & 0.395 & & & 0.500 & & & 0.500 \\
\hline Poorly & 40 & 32 & 8 & & 31 & 9 & & 33 & 7 & \\
\hline Highly and moderately & 40 & 30 & 10 & & 32 & 8 & & 34 & 6 & \\
\hline
\end{tabular}

${ }^{\mathrm{a}} \mathrm{P}<0.05$, determined using a $\chi^{2}$ test. MMP, matrix metalloproteinase.

were significantly higher $(\mathrm{P}<0.05)$. Therefore, these results show that expression levels of MMP-1 and MMP-10 were significantly increased in gastric cancer and particularly in metastatic gastric cancer.

$H$. pylori infection rate in gastric cancer is higher than that in chronic gastritis. To detect the H. pylori infection level in gastric cancer and chronic gastritis specimens, fasting blood was collected and $H$. pylori IgG levels were measured by ELISA. There were 62 cases of $H$. pylori infection out of 80 gastric cancer cases with a positive rate of $77.5 \%$, and 13 cases of $H$. pylori infection out of 40 chronic gastritis cases with a positive rate of $32.5 \%$. Significantly higher levels of H. pylori infection were observed in the gastric cancer specimens than those in the chronic gastritis specimens $(\mathrm{P}<0.05$, data not shown). This result suggests that there was significant $H$. pylori infection in gastric cancer.

Correlation between H. pylori infection, MMP-1 and MMP-10 protein expression and gastric cancer pathological characteristics. To determine the roles of $H$. pylori infection and MMP-1 and MMP-10 protein expression in gastric cancer, the association between these factors and the clinical pathological characteristics of gastric cancer were analyzed. As shown in Table II, positive $H$. pylori infection and positive expression of MMP-1 and MMP-10 were associated with lymph node metastasis and clinical stage $(\mathrm{P}<0.05)$, but not with age, gender or differentiation degree $(\mathrm{P}>0.05)$. To further analyze the associations among $H$. pylori infection and MMP-1 and MMP-10 expression, correlation analyses were performed.
It was revealed that MMP-1 and MMP-10 expression was significantly positively correlated in gastric cancer $(\mathrm{r}=0.8321$, $\mathrm{P}<0.05$; data not shown). H. pylori infection and MMP-1 and MMP-10 expression were also significantly positively correlated $(\mathrm{r}=0.8718, \mathrm{P}<0.05$ and $\mathrm{r}=0.5477, \mathrm{P}<0.05$, respectively; data not shown). Thus, these data suggest that associations exist among H. pylori infection, MMP-1 expression and MMP-10 expression in the development and metastasis of gastric cancer.

MMP-1 and MMP-10 expression in H.pylori-infected gastric cancer cells is upregulated. To investigate the effect of H. pylori infection on MMP-1 and MMP-10 expression, the expression levels of MMP-1 and MMP-10 were detected in MGC-803 cells following H.pylori infection. Western blotting was conducted at $6,12,24$ and $48 \mathrm{~h}$ of infection. The results for MMP-1 and MMP-10 are shown in Figs. 2 and 3, respectively. As shown in Fig. 2, at $6 \mathrm{~h}$ of H.pylori infection, the intracellular MMP-1 expression level was not significantly changed, while at $12 \mathrm{~h}$ of $H$. pylori infection, the intracellular MMP-1 expression was significantly increased compared with that in the uninfected group at $0 \mathrm{~h}(\mathrm{P}<0.05)$. MMP-1 expression at 24 and $48 \mathrm{~h}$ was maintained at a similar level to that at $12 \mathrm{~h}$, which was also significantly increased compared with expression in the uninfected group at $0 \mathrm{~h}(\mathrm{P}<0.05)$. However, the intracellular MMP-10 expression was significantly increased at $6 \mathrm{~h}$ of $H$. pylori infection compared with that in the uninfected group at $0 \mathrm{~h}(\mathrm{P}<0.05)$ (Fig. 3). Similarly, MMP-10 expression at 12,24 and $48 \mathrm{~h}$ of $H$. pylori infection remained significantly increased $(\mathrm{P}<0.05)$. These results indicate that $H$. pylori 


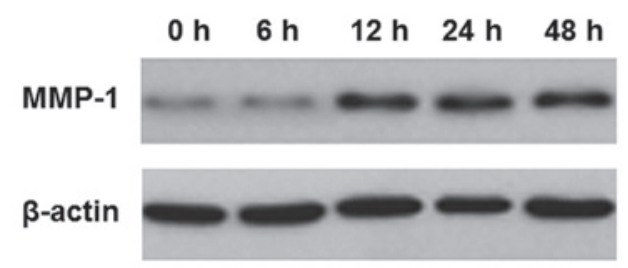

B

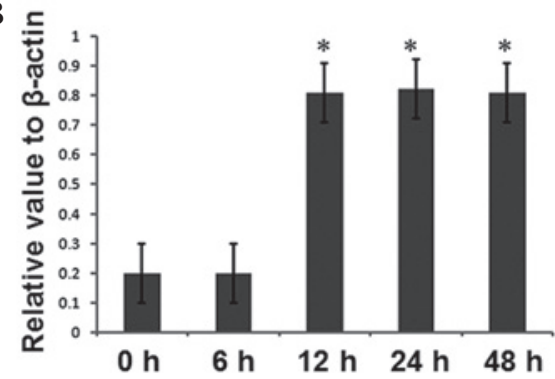

Figure 2. MMP-1 protein expression following Helicobacter pylori infection in MGC-803 gastric cancer cells. MMP-1 expression was detected by western blotting at $0,6,12,24$ and $48 \mathrm{~h}$ after $H$. pylori infection. (A) Representative western blotting results. (B) Quantitative western blotting results. ${ }^{*} \mathrm{P}<0.05$ compared with the value at $0 \mathrm{~h}$. MMP-1, matrix metalloproteinase-1.

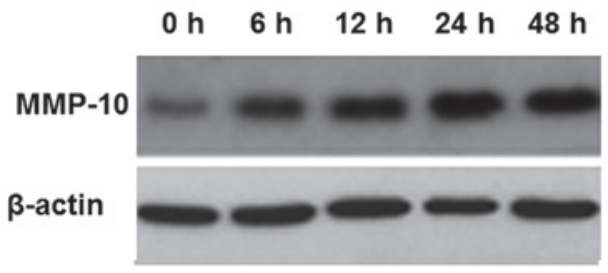

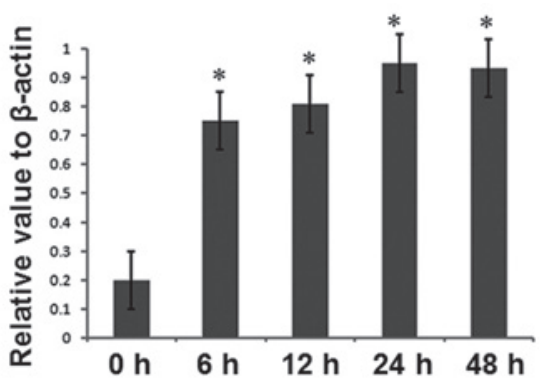

Figure 3. MMP-10 protein expression following Helicobacter pylori infection in MGC-803 gastric cancer cells. MMP-10 expression was detected by western blotting at $0,6,12,24$ and $48 \mathrm{~h}$ after $\mathrm{H}$. pylori infection. (A) Representative western blotting results. (B) Quantitative western blotting results. ${ }^{*} \mathrm{P}<0.05$ compared with the value at $0 \mathrm{~h}$. MMP-10, matrix metalloproteinase- 10 .

infection promoted the expression of MMP-1 and MMP-10 in gastric cancer cells.

\section{Discussion}

MMP-1 is predominantly involved in the degradation of stromal components (10), and is important in tumor invasion and metastasis (11). MMP-10 is considered an important factor in the activation of other MMP (such as MMP-1) precursors in human tumor cells (12). In addition, MMP-10 can degrade proteins, including collagen III/IV/V, gelatin, nidogen, laminin-l and proteoglycans, thereby contributing to metastasis (13). This study has shown that MMP-1 and MMP-10 expression was higher in patients with gastric cancer with metastasis than in those with gastric cancer without metastasis. MMP-1 and MMP-10 expression in patients with gastric cancer and in those with metastatic gastric cancer was significantly higher than that in patients with gastritis. The positive expression of MMP-1 and MMP-10 was associated with lymph node metastasis and clinical stage, but not with age, gender or differentiation degree, indicating that MMP-1 and MMP-10 expression was involved in gastric cancer metastasis. It was also found that MMP-1 expression was correlated with MMP-10 expression, indicating that these two different types of MMPs may play a synergic role in gastric cancer genesis, invasion and metastasis. Thus, the simultaneous detection of MMP-1 and MMP-10 may be important in the evaluation of the prognosis and metastasis of gastric cancer.

Crawford et al (14) revealed that MMP-7 levels were significantly increased in H. pylori-Cag-positive gastric cancer cells, while not significantly increased in uninfected cells. Gööz et al (15) found that $H$. pylori can stimulate the secretion of MMP-1, MMP-3 and TIMP-3 in gastric cancer cells in vitro. However, TIMP-2 expression levels were not significantly changed. In the present study, it was revealed that H. pylori infection was significantly associated with MMP-1 and MMP-10 expression. Positive H.pylori infection was also associated with lymph node metastasis and clinical stage, and the number of specimens positive for $H$. pylori infection in advanced gastric cancer was significantly higher than that in early stage disease. This may be a result of the destruction of the normal mucosal barrier following $H$. pylori infection, stimulating MMP-1 and MMP-10 expression. MMPs can destroy the matrix-degrading balance, promote cancer invasion through the histological barrier (constituted by the basement membrane and ECM) into the surrounding tissues and metastasis to distant tissues, causing gastric cancer metastasis.

The present data showed that co-culturing $H$. pylori with MGC-803 cells led to the secretion of MMP-1 and MMP-10 by the MGC-803 cells, indicating that $H$. pylori infection may be involved in metastasis through the upregulation of MMP-1 and MMP-10 expression. At $6 \mathrm{~h}$ after H. pylori infection, MMP-10 expression was upregulated, while at $12 \mathrm{~h} \mathrm{MMP-1}$ began to be expressed. MMP-1 and MMP-10 expression was maintained at a high level at 24 and $48 \mathrm{~h}$ after $H$. pylori infection. As shown in previous studies $(12,16)$, MMP-10 can activate precursors of other MMPs (including MMP-1). In this study, the results indicate that in the H.pylori-induced MMP secretion in gastric cancer cells, MMP-10 may be involved in the activation of MMP-1.

In conclusion, $H$. pylori infection is not only the primary factor of gastric cancer that causes mucosal injury, promotes 
tumor suppressor gene mutation and activates oncogenes, but may also be involved in invasion and metastasis by promoting MMP secretion, leading to gastric cancer deterioration and a decrease in survival time. H. pylori infection may be involved in gastric cancer metastasis through the mechanism of upregulating the expression of MMP-1 and MMP-10.

\section{Acknowledgements}

This study was supported by the Project of Hunan Provincial Education Department (grant no. 09A077).

\section{References}

1. Piazuelo MB, Epplein M and Correa P: Gastric cancer: an infectious disease. Infect Dis Clin North Am 24: 853-869, 2010.

2. Pizzi M, Saraggi D, Fassan M, Megraud F, Di Mario F and Rugge M: Secondary prevention of epidemic gastric cancer in the model of Helicobacter pylori-associated gastritis. Dig Dis 32: 265-274, 2014

3. Pandey R, Misra V, Misra SP, Dwivedi M, Kumar A and Tiwari BK: Helicobacter pylori and gastric cancer. Asian Pac J Cancer Prev 11: 583-588, 2010.

4. Borlace GN, Butler RN and Brooks DA: Monocyte and macrophage killing of Helicobacter pylori: relationship to bacterial virulence factors. Helicobacter 13: 380-387, 2008

5. Zhang Q, Li Y, Li X, Zhou W, Shi B, Chen H and Yuan W: PARP-1 Val762Ala polymorphism, $\mathrm{CagA}^{+} H$. pylori infection and risk for gastric cancer in Han Chinese population. Mol Biol Rep 36: 1461-1467, 2009.

6. Rydlova M, Holubec L Jr, Ludvikova M Jr, et al: Biological activity and clinical implications of the matrix metalloproteinases. Anticancer Res 28: 1389-1397, 2008.

7. Scherer RL, McIntyre JO and Matrisian LM: Imaging matrix metalloproteinases in cancer. Cancer Metastasis Rev 27 : 679-690, 2008
8. Orlichenko LS and Radisky DC: Matrix metalloproteinases stimulate epithelial-mesenchymal transition during tumor development. Clin Exp Metastasis 25: 593-600, 2008.

9. Gentner B, Wein A, Croner RS, et al: Differences in the gene expression profile of matrix metalloproteinases (MMPs) and their inhibitors (TIMPs) in primary colorectal tumors and their synchronous liver metastases. Anticancer Res 29: 67-74, 2009.

10. Shim KN, Jung SA, Joo YH and Yoo K: Clinical significance of tissue levels of matrix metalloproteinases and tissue inhibitors of metalloproteinases in gastric cancer. J Gastroenterol 42: 120-128, 2007.

11. Fujimoto D, Hirono Y, Goi T, Katayama K and Yamaguchi A: Prognostic value of protease-activated receptor-1 (PAR-1) and matrix metalloproteinase-1 (MMP-1) in gastric cancer. Anticancer Res 28: 847-854, 2008.

12. Frederick LA, Matthews JA, Jamieson L, et al: Matrix metalloproteinase-10 is a critical effector of protein kinase Ciota-Par6alpha-mediated lung cancer. Oncogene 27: 4841-4853, 2008.

13. Dannewitz B, Edrich C, Tomakidi P, et al: Elevated gene expression of MMP-1, MMP-10, and TIMP-1 reveal changes of molecules involved in turn-over of extracellular matrix in cyclosporine-induced gingival overgrowth. Cell Tissue Res 325: 513-522, 2006

14. Crawford HC, Krishna US, Israel DA, et al: Helicobacter pylori strain-selective induction of matrix metalloproteinase-7 in vitro and within gastric mucosa. Gastroenterology 125: 1125-1136, 2003.

15. Gööz M, Shaker M, Gööz P and Smolka AJ: Interleukin 1beta induces gastric epithelial cell matrix metalloproteinase secretion and activation during Helicobacter pylori infection. Gut 52: 1250-1256, 2003.

16. Nakamura H, Fujii Y, Ohuchi E, Yamamoto E and Okada Y: Activation of the precursor of human stromelysin 2 and its interactions with other matrix metalloproteinases. Eur J Biochem 253: 67-75, 1998 . 